\title{
Cold plasma as a potential adjunctive therapy in COVID-19: report of three cases.
}

\author{
Fahimeh Abdollahimajd ${ }^{1,2}$, Mohammad Reza Pourani ${ }^{1}$, Alireza Fatemi ${ }^{3}$, and Hamideh \\ Moravvej $^{1}$ \\ ${ }^{1}$ Skin Research Center, Shahid Beheshti University of Medical Sciences \\ ${ }^{2}$ Clinical Research Development Unit, Shohada-e Tajrish Hospital, Shahid Beheshti \\ University of Medical Sciences \\ ${ }^{3}$ Men's Health and Reproductive Health Research Center, Shahid Beheshti University of \\ Medical Sciences
}

January 4, 2021

\section{Cold plasma as a potential adjunctive therapy in COVID-19: report of three cases}

Fahimeh Abdollahimajd ${ }^{1,2}$, Mohammad Reza Pourani ${ }^{1}$, Alireza Fatemi ${ }^{3}{ }^{\text {Hamideh Moravvej }}{ }^{{ }^{*}}$

${ }^{1}$ Skin Research Center, Shahid Beheshti University of Medical Sciences, Tehran, Iran;

${ }^{2}$ Clinical Research Development Unit, Shohada-e Tajrish Hospital, Shahid Beheshti University of Medical Sciences, Tehran, Iran

${ }^{3}$ Men's Health and Reproductive Health Research Center, Shahid Beheshti University of Medical Sciences, Tehran, Iran

*Corresponding author: Hamideh Moravvej, Skin Research Center, Shohada-e Tajrish Hospital, Shahid Beheshti University of Medical Sciences, Tehran, Iran; Tel: +98- 21-22741507, Fax:+98-2122744392, Email: hamidehmoravej@sbmu.ac.ir

* Co-corresponding author: Mohammad Reza Pourani, Skin Research Center, Shohada-e Tajrish Hospital, Shahid Beheshti University of Medical Sciences, Tehran, Iran; Tel: +98- 21-22741507, Fax:+982122744392, Email: mreza.pourani92@gmail.com

Word count: 595 Number of references: 5Items: 2 Figures

Keywords: Cold plasma, COVID-19, Coronavirus 2019, Treatment

Running title: COVID-19 infection and cold plasma treatment

Funding: This research did not receive any specific grant from funding agencies in the public, commercial, or not-for-profit sectors.

Competing Interests: The authors declare no conflict of interest.

Cold plasma as a potential adjunctive therapy in COVID-19: report of three cases

\section{Abstract:}

During the COVID-19 pandemic many researchers have examined some potential treatments to battle against a Severe Acute Respiratory Syndrome Coronavirus 2 (SARS-CoV-2). Immediate assessment of suggested treatments may lead to decreased in burden of SARS-CoV-2 infection. Plasma medicine is a novel field of 
therapy that through different mechanisms causes anti-microbial impacts. Recent studies have demonstrated favourable anti-viral effects of plasma therapy. Current researchers recommended evaluating cold plasma as a possible therapy against COVID-19 infection. In this survey, we examined cold plasma application in three patients with COVID-19 infection.

Keywords: Cold plasma, COVID-19, Coronavirus 2019, Treatment

\section{Dear editor}

Many people throughout the world have contended with a Severe Acute Respiratory Syndrome Coronavirus 2 (SARS-CoV-2) pandemic. Researchers have investigated some potential therapies to exploit against coronavirus disease 2019 (COVID-19). ${ }^{1}$ Plasma medicine is a novel field of treatment that has broad anti-microbial effects. Cold plasma has been employed in this field to inactivate virus functions. ${ }^{2,3}$ Recent studies have described potential efficacy of cold plasma against different viruses. ${ }^{2}$ Herein, we reported three patients with COVID-19 infection who were treated with cold plasma and demonstrated a significant response. Cold plasma (Cloudburst, Tose'eMadare Hiro Co, Iran) was applied in each naris for 5seconds and in oropharynx for 10 seconds for at least three consecutive days (Figure 1 )

Case 1. A 32-year-old male presented with fever, chills and headache was referred for evaluation of COVID19; the result of nasopharyngeal PCR test was positive. His symptoms were aggravated and he had experienced malaise, myalgia, diarrhea, dry cough and anosmia. Consequently, a low-dose spiral lung computed tomography (CT) was requested that showed bilateral patchy infiltrations compatible with COVID-19 (Figure 2 a,b ). He was recommended to keep home quarantine. On the $14^{\text {th }}$ day of the onset of symptoms, he was treated with nasal cold plasma and his symptoms improved significantly. His anosmia on the day of treatment and other symptoms gradually alleviated on the following days (Figure $\mathbf{2} \mathbf{c}, \mathbf{d}$ ).

Case 2 . A 33-year-old male patient was referred to our clinic with fever $39^{\circ} \mathrm{C}$, headache, dry cough, myalgia, diarrhea and malaise. We requested a nasopharyngeal PCR test for COVID-19 that was positive; spiral lung CT scan was also compatible with COVID-19. During the disease course, he experienced anosmia and also ageusia. We recommended keeping self-isolation. Due to the persistence of symptoms, this patient was also treated with nasal cold plasma on the $10^{\text {th }}$ day. His symptoms especially anosmia diminished notably.

Case 3. A 38-year-old male patient with low grade fever, dry cough, myalgia and anosmia was referred for nasopharyngeal PCR test for COVID-19 that was positive. Patient had been treated with azithromycin and naproxen for 6days. On the $7^{\text {th }}$ day, nasal cold plasma was administered daily for three days. Initially, he was experienced exacerbation of dry cough after nasal cold plasma application, probably due to the ozone gas effects. However, anosmia was palliated on the first day of treatment significantly and other symptoms were relieved gradually until the third day of cold plasma therapy.

During the COVID-19 pandemic, many investigators are assessing new treatments against SARS-CoV-2. Cold plasma is a new field of therapy in infectious diseases. ${ }^{1}$ The most important components of cold plasma for its antimicrobial characteristics are UV radiation and reactive oxygen and/or nitrogen species that can damage nucleic acids. ${ }^{2}$ Recent studies have reported cold plasma as a potential treatment for enteric viruses like norovirus, and adenovirus as well as airborne respiratory viruses, such as respiratory syncytial virus, and Influenza A(H5N2) ${ }^{2-4}$ It has been showed that cold plasma also could inactivate viruses through water decontamination. ${ }^{2}$ Recent studies investigated cold plasma as a candidate treatment for COVID-19 infection. ${ }^{1,5}$

In all of our patients after using nasal cold plasma, their symptoms and general condition gradually improved; however, they were experienced mild symptoms of COVID-19 infection. To the best of our knowledge, this is the first report of using cold plasma as an adjunctive treatment for COVID-19 infection. Cold plasma may be suggested as a potential adjunctive therapy for treatment of patients with COVID-19 under mechanical 
ventilation to prevent upper respiratory infections. However, more clinical investigations are demanded to evaluate the efficacy and safety of cold plasma in treatment of SARS-CoV-2 infection.

Funding: This research did not receive any specific grant from funding agencies in the public, commercial, or not-for-profit sectors.

Competing interests: The authors declare no conflict of interest.

Acknowledgments: We thank the patients and their families for the willingness to provide these images. We also thank all the members of healthcare team who are involved in the care of COVID-19 patients. The patients in this manuscript have given written informed consent to publication of their case details.

\section{References:}

1. Wang S, Liu W, Song Y, Xia T, Lu X, Song L et al (2020) Non-Drug Therapy to Combat Coronavirus. Open Journal of Regenerative Medicine 9(02):65

2. Filipić A, Gutierrez-Aguirre I, Primc G, Mozetič M, Dobnik D (2020) Cold plasma, a new hope in the field of virus inactivation. Trends in Biotechnology. DOI: 10.1016/j.tibtech.2020.04.003

3. Bernhardt T, Semmler ML, Schäfer M, Bekeschus S, Emmert S, Boeckmann L (2019) Plasma medicine: Applications of cold atmospheric pressure plasma in dermatology. Oxidative medicine and cellular longevity 2019:1-10

4. Pradeep P, Chulkyoon M (2016) Non-thermal plasmas (NTPs) for inactivation of viruses in abiotic environment. Research Journal of Biotechnology 11(6):91-96

5. Ghernaout D, Elboughdiri N (2020) Disinfecting Water: Plasma Discharge for Removing Coronaviruses. Open Access Library Journal 7(4):1-29

\section{Figure Legends:}

Figure 1: Cold plasma application

Figure 2: Bilateral patchy infiltrations compatible with COVID-19 (a, b). No sign of COVID-19 is seen after treatment $(\mathrm{c}, \mathrm{d})$ 